\title{
Renormalization of entanglement entropy from topological terms
}

\author{
Giorgos Anastasiou, Ignacio J. Araya, and Rodrigo Olea \\ Departamento de Ciencias Físicas, Universidad Andres Bello, Sazié 2212, Piso 7, Santiago, Chile
}

(Received 24 February 2018; published 16 May 2018)

\begin{abstract}
We propose a renormalization scheme for entanglement entropy of three-dimensional CFTs with a four-dimensional asymptotically AdS gravity dual in the context of the gauge/gravity correspondence. The procedure consists in adding the Chern form as a boundary term to the area functional of the Ryu-Takayanagi minimal surface. We provide an explicit prescription for the renormalized entanglement entropy, which is derived via the replica trick. This is achieved by considering a Euclidean gravitational action renormalized by the addition of the Chern form at the spacetime boundary, evaluated in the conically-singular replica manifold. We show that the addition of this boundary term cancels the divergent part of the entanglement entropy, recovering the results obtained by Taylor and Woodhead. We comment on how this prescription for renormalizing the entanglement entropy is in line with the general program of topological renormalization in asymptotically AdS gravity.
\end{abstract}

DOI: $10.1103 /$ PhysRevD.97.106011

\section{INTRODUCTION}

In the context of the AdS/CFT correspondence [1-3], the entanglement entropy (EE) of an entangling region $A$ in a CFT with an asymptotically AdS (AAdS) Einstein gravity dual, can be computed as the volume of a codimension-2 minimal surface. In particular, this is achieved by calculating the volume of the minimal surface $\Sigma$ in the bulk whose boundary $\partial \Sigma$ is conformal to the entangling surface $\partial A$, which bounds $A$ at the conformal boundary $C$. This proposal is referred to as the Ryu-Takayanagi (RT) prescription $[4,5]$. In order to illustrate the different submanifolds involved in this construction, and the geometric relations between them, we include a schematic diagram in Fig. 1.

This definition for the $\mathrm{EE}$ is formally divergent, due to the presence of an infinite conformal factor at the AdS boundary $B$, what is manifest in the Fefferman-Graham (FG) form of the metric [6,7]. As it was shown by Taylor and Woodhead [8], it is possible to renormalize the EE by adding counterterms constructed through the Replica Trick [8-10] from the standard Holographic Renormalization procedure [11-17]. This is done by evaluating the usual counterterms for Einstein gravity at the conically singular spacetime boundary, which is conformal to the manifold of the Replica CFT.

Published by the American Physical Society under the terms of the Creative Commons Attribution 4.0 International license. Further distribution of this work must maintain attribution to the author(s) and the published article's title, journal citation, and DOI. Funded by SCOAP ${ }^{3}$.
Here, we propose an alternative regularization prescription that has the advantage of giving the countertem for the EE as a single boundary term, which can be written in closed form for CFTs of arbitrary (odd) dimensions that have an (evendimensional) AAdS E-H gravity dual. This boundary term corresponds to the Chern form evaluated at the boundary of the RT minimal surface, which is conformal to the entangling surface that bounds the entangling region in the CFT. In particular, we propose that the renormalized $\mathrm{EE}$ of a

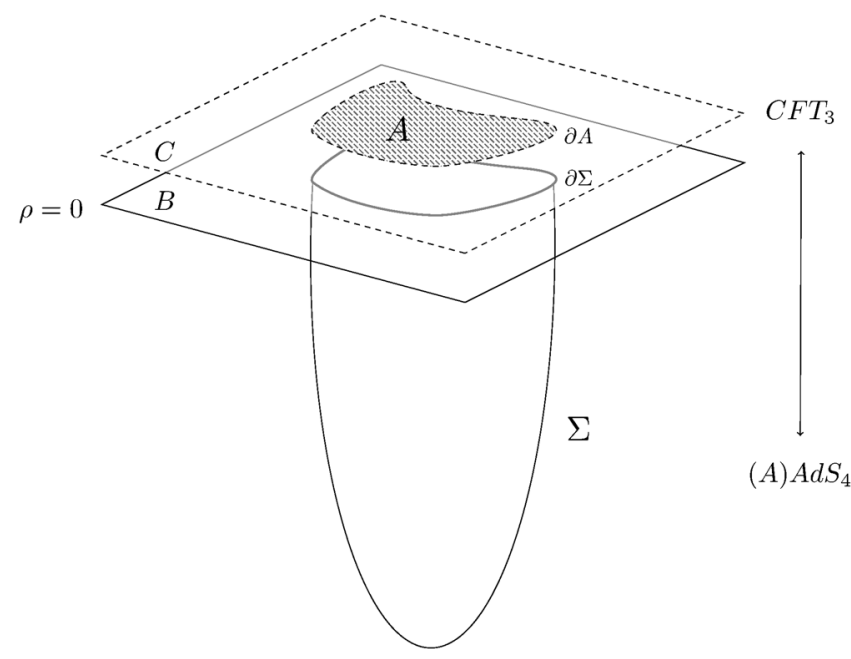

FIG. 1. In this diagram, we show all the submanifolds involved in the RT construction. On the field theory side, $C$ is the conformal boundary where the CFT is defined, $A$ is the entangling region and $\partial A$ is the entangling surface. On the gravity side, $B$ is the boundary of spacetime, $\Sigma$ is the minimal surface in the bulk and $\partial \Sigma$ is its border at the spacetime boundary. Both sides are related such that $C$ is conformal to $B$ and $\partial A$ is conformal to $\partial \Sigma$. 
three-dimensional CFT with a four-dimensional AAdS E-H gravity dual is given by

$$
S_{\mathrm{EE}}^{\mathrm{ren}}=\frac{\operatorname{Vol}(\Sigma)}{4 G}+\frac{\ell^{2}}{8 G} \int_{\partial \Sigma} B_{1},
$$

where $\Sigma$ is the codimension-2 RT minimal surface, $\partial \Sigma$ is its border at the spacetime boundary $B, \ell$ is the AdS radius. In addition, $B_{1}$ is the first Chern form evaluated at the border of the RT minimal surface, whose detailed form is given in Eq. (33). Therefore, we show that the EE counterterm $\left(S_{\mathrm{EE}}^{c t}\right)$ is given by the $B_{1}$ term, which depends on the induced metric $\tilde{\gamma}$ of $\partial \Sigma$, and on its extrinsic curvature with respect to the radial foliation along the holographic radial coordinate $\rho$, which is the parameter of the FG expansion. It is apparent then, that the Chern form is written in terms of both intrinsic and extrinsic quantities of $\partial \Sigma$. The particular features of $S_{\mathrm{EE}}^{c t}$ are explained in Sec. III.

In order to obtain the EE boundary counterterm, we consider the Replica Trick, where the conically singular replica manifold is constructed as described in [18]. We also consider the gravitational Euclidean action in the AAdS bulk. The EE is then expressed in terms of a derivative of said action evaluated on the replica manifold, with respect to the conical angular parameter. Therefore, if the action is itself renormalized, the EE computed in this manner will be renormalized as well. In order to renormalize the bulk gravitational action, we consider the Kounterterms proposal [19-23], instead of the standard Holographic Renormalization prescription [11-17]. This choice is made because of the fact that in the Kounterterms scheme, the counterterm that renormalizes the on-shell gravitational action can be written in closed form, as a single boundary term with topological origin. As a matter of fact, this prescription is known for arbitrary dimensions and also for any gravity theory of Lovelock type. For the evaluation of the renormalized action on the replica manifold, we consider a generalization of the Euler theorem to conically singular manifolds in four-dimensions, derived by using distributional geometry [24,25]. Thus, the counterterm of the action splits into a regular part at the spacetime boundary and another at $\partial \Sigma$. The latter results in a contribution proportional to the angular parameter which gives the $B_{1}$ piece of Eq. (1). Upon taking the derivative of the action with respect to the conically singular parameter, we obtain $S_{\mathrm{EE}}^{\text {ren }}$ as shown in Eq. (1), where the bulk part of the action gives the usual RT term. We emphasize that the form of $S_{\mathrm{EE}}^{\text {ren }}$ obtained in the $\mathrm{AdS}_{4} / \mathrm{CFT}_{3}$ case, and shown in Eq. (1), is equivalent to the known result of

$$
S_{\mathrm{EE}}^{\mathrm{ren}}=\frac{\operatorname{Vol}(\Sigma)}{4 G}-\frac{\ell}{4 G} \int_{\partial \Sigma} d x \sqrt{\tilde{\gamma}}
$$

given in [8], as explained in Sec. III B.
This paper is organized as follows: In Sec. II, we explain the setup used for obtaining the renormalized EE. We give a general overview of the definition of EE and of the Replica Trick, applied to the AdS/CFT context. We then explain the generalization of the Euler theorem for conically singular manifolds, and in particular, for the case without a $U(1)$ isometry in four dimensions. Then, we introduce the renormalized Euclidean gravitational action obtained by the Kounterterms procedure. In Sec. III, we use the elements described in the setup to obtain the $S_{\mathrm{EE}}^{c t}$ in the $\mathrm{AdS}_{4} / \mathrm{CFT}_{3}$ case. We also expand the obtained boundary counterterm considering the explicit covariant embedding of the $\Sigma$ minimal surface on the bulk. When taking the FG expansion of its induced metric we show that $S_{\mathrm{EE}}^{c t}$ can be re-written in the standard way of Eq. (2). We explicitly check the finiteness of $S_{\mathrm{EE}}^{\text {ren }}$, and we verify that the standard computation of the renormalized $\mathrm{EE}$ of a disclike entangling region in $\mathrm{CFT}_{3}$ is correctly recovered. We also give a new interpretation of $S_{\mathrm{EE}}^{\text {ren }}$ in terms of the topological and geometrical properties of the minimal surface $\Sigma$ as an AAdS submanifold [see Eq. (47)]. Finally, in Sec. IV, we give a general outlook of the method and comment on possible generalizations thereof.

\section{THE SETUP: REPLICA TRICK AND RENORMALIZED EUCLIDEAN ACTION IN THE CONICALLY SINGULAR MANIFOLD}

We proceed to explain the different elements of the setup considered in order to obtain the renormalized entanglement entropy $S_{\mathrm{EE}}^{\mathrm{ren}}$. We start by giving a brief overview of $\mathrm{EE}$ in the AdS/CFT context, discussing how to compute it with the replica trick, in terms of derivatives of the on-shell Euclidean action. Then, we explain the generalization of the Euler theorem to four-dimensional conically singular manifolds without $U(1)$ isometry. Finally, we consider the renormalized Euclidean gravitational E-H action, as obtained by the Kounterterms procedure, and comment on its properties and usefulness for the computation of $S_{\mathrm{EE}}^{\text {ren }}$.

\section{A. Entanglement entropy and replica trick}

The EE $[4,5,9,10,26,27]$ is defined as the von Neumann entropy of the reduced density matrix of a quantum subsystem $A$, i.e.

$$
S_{\mathrm{EE}}=-\operatorname{Tr}\left(\hat{\rho}_{A} \ln \hat{\rho}_{A}\right),
$$

and it encodes the degree of entanglement of the subsystem $A$ with the rest of the system $\left(A^{c}\right)$. The first proposal for computing EEs of CFTs in the AdS/CFT framework was the RT formula [4]. Said formula states that the EE of an entangling region $A$ in a (D-1)-dimensional CFT with a D-dimensional AAdS gravity dual is equal to the volume of a codimension 2 minimal hypersurface $(\Sigma)$ in the AAdS bulk whose border is conformal to the one of the entangling 
region $(A)$ at the conformal boundary; i.e., $S_{\mathrm{EE}}=\frac{\operatorname{Vol}(\Sigma)}{4 G}$ (in natural units). This formula is analogous to the BekensteinHawking entropy formula for a black hole [28-30], and it was shown (e.g., by Lewkowycz and Maldacena in [10]) that indeed both formulas can be obtained from the replica trick [8-10].

The computation of the EE by the replica trick considers that Eq. (3) can be rewritten as

$$
S_{\mathrm{EE}}=\lim _{n \rightarrow 1}-\frac{1}{n-1} \ln \left(\operatorname{Tr}\left(\hat{\rho}_{A}^{n}\right)\right),
$$

and therefore, the EE is expressed in terms of the trace of the $n$th power of the reduced density matrix. In order to compute this trace, one constructs a branched cover of the conformal boundary $C$ where the CFT is defined. This is achieved by gluing together $\mathrm{n}$ copies of the original (Euclideanized) boundary with a cut along the entangling region whose EE is being computed [18]. The gluing is done such that, when defining an angular coordinate that circles around the border of the entangling region, after $2 \pi$ rotations along the coordinate, the copies are cyclically permuted. Labelling this branched cover manifold as $C_{n}$, one realizes that it has a $Z_{n}$ symmetry corresponding to cycling from one copy (replica) of the CFT to another. Finally, one defines the orbifold $\hat{C}_{n}$ as the quotient of the cover manifold by the permutation symmetry, i.e., $\hat{C}_{n}=C_{n} / Z_{n}$. The orbifold $\hat{C}_{n}$ is conically singular, with an opening angle of $\frac{2 \pi}{n}$. However, because the permutation symmetry is the discrete symmetry $Z_{n}$, the orbifold does not have a $U(1)$ isometry in general.

Considering the $\hat{C}_{n}$ orbifold, $\operatorname{Tr}\left(\hat{\rho}_{A}^{n}\right)$ can be computed in terms of the partition function of the replica CFT defined on the orbifold, as

$$
\operatorname{Tr}\left(\hat{\rho}_{A}^{n}\right)=n\left(\ln \left(Z\left(\hat{C}_{n}\right)\right)-\ln \left(Z\left(\hat{C}_{1}\right)\right)\right),
$$

where $\hat{C}_{1}$ (which is equal to $C$ ) is the manifold of the original CFT and $Z\left(\hat{C}_{1}\right)$ is its partition function.

One then defines the orbifold $\hat{M}_{n}$ as the extension of $\hat{C}_{n}$ into the AAdS bulk, by requiring the bulk metric to be a solution of the equations of motion. Because the orbifold $\hat{M}_{n}$ is a solution in the bulk, the semiclassical approximation can be used to write the partition functions in Eq. (5) in terms of the corresponding gravitational Euclidean on-shell actions in the AAdS bulk (including boundary terms). Then, in the saddle-point approximation, one has that $\ln \left(Z\left(\hat{C}_{n}\right)\right)=-I_{E}\left(\hat{M}_{n}\right)$, and therefore,

$$
\operatorname{Tr}\left(\hat{\rho}_{A}^{n}\right)=-n\left(I_{E}\left(\hat{M}_{n}\right)-I_{E}\left(\hat{M}_{1}\right)\right) .
$$

Thus, in the AdS/CFT context, the EE computed by the replica trick can be written as

$$
S_{\mathrm{EE}}=\lim _{n \rightarrow 1} \frac{n}{n-1}\left(I_{E}\left(\hat{M}_{n}\right)-I_{E}\left(\hat{M}_{1}\right)\right)=\left.n^{2} \partial_{n} I_{E}\left(\hat{M}_{n}\right)\right|_{n=1} .
$$

Finally, for ease of computation, we define the angular parameter $\alpha$ such that $\alpha=\frac{1}{n}$, where the cone then has an angular deficit given by $2 \pi(1-\alpha)=2 \pi\left(1-\frac{1}{n}\right)$. Thus, in the case of a three-dimensional CFT with a four-dimensional AAdS gravity dual, the EE is

$$
S_{\mathrm{EE}}=-\left.\partial_{\alpha} I_{E}\left(\hat{M}_{4}^{(\alpha)}\right)\right|_{\alpha=1},
$$

where now $\hat{M}_{4}^{(\alpha)}$ denotes the four-dimensional orbifold with angular deficit given by $2 \pi(1-\alpha)$.

In order to evaluate this Euclidean action, we first need to discuss some properties of differential geometry in conically singular manifolds $[24,25,31,32]$. In particular, in the next section, we review a generalization of the Euler theorem for squashed cones (conically singular manifolds without $U(1)$ isometry) in four dimensions.

\section{B. Euler theorem for conically singular manifolds in four dimensions}

In differential geometry, topological invariants are interesting because they characterize properties of manifolds that are robust under continuous deformations of their metric. For example, the Euler characteristic in $D=2 m$ can be written as the integral of a precise combination of a product of $m$-curvature terms, with the addition of the $m$ th Chern form in a manifold with boundaries. The Chern form is expressible considering both intrinsic and extrinsic curvatures of the boundary's induced metric. Therefore, this way of writing the Euler characteristic provides a global relation between the curvature of a bulk manifold, and the curvatures of its boundary. In particular, the Euler theorem [19], which is valid for $2 m$-dimensional manifolds, states that

$$
\int_{M_{2 m}} \varepsilon_{2 m}=(4 \pi)^{m} m ! \chi\left(M_{2 m}\right)+\int_{\partial M_{2 m}} B_{2 m-1},
$$

where $\varepsilon_{2 m}$ is the Euler density in $2 m$ dimensions, $\chi\left(M_{2 m}\right)$ is the Euler characteristic of the manifold $M_{2 m}$, and $B_{2 m-1}$ is the $m$ th Chern form at the boundary of the manifold. In the particular case of $m=2$, and therefore $\operatorname{dim}\left(M_{2 m}\right)=4$, the Euler density $\varepsilon_{4}$ is the usual Gauss-Bonnet term and $B_{3}$ is the second Chern form (given in Eq. (20) in Gauss normal coordinates).

As we will see in Sec. III, in order to obtain the renormalized version of $S_{\mathrm{EE}}$, we need to evaluate either $\varepsilon_{4}$ or $B_{3}$ on conically singular manifolds (without $U(1)$ rotational isometry). To this end, we consider the results obtained by Fursaev, Patrushev and Solodukhin (FPS) [25] regarding 
the computation of quadratic terms in the curvature, for conically singular manifolds in four dimensions.

In order to compute the integral of the quadratic terms, which correspond to the Ricci scalar squared, the Ricci tensor squared and the Riemann tensor squared, FPS used the methods of distributional geometry, as described in $[24,25]$. There, a conically singular orbifold was considered as the limit of a sequence of regular manifolds whose metrics are parametrized by a certain regularization parameter. Then, the quadratic terms are computed, and the parameter is taken to zero, in order to recover the conically singular manifold (for further details, we refer the reader to the original papers).

In particular, FPS obtained that the integral of the square of the Riemann tensor evaluated on the four-dimensional orbifold $\hat{M}_{4}^{(\alpha)}$ is given by

$$
\begin{aligned}
& \int_{\hat{M}_{4}^{(\alpha)}} d^{4} x \sqrt{G}\left(R^{(\alpha)}\right)_{\nu \sigma \lambda}^{\mu}\left(R^{(\alpha)}\right)_{\mu}^{\nu \sigma \lambda} \\
& =\int_{M_{4}} d^{4} x \sqrt{G}\left(R^{(r)}\right)_{\nu \sigma \lambda}^{\mu}\left(R^{(r)}\right)_{\mu}^{\nu \sigma \lambda}+8 \pi(1-\alpha) \\
& \quad \times \int_{\Sigma} d^{2} x \sqrt{\gamma}\left(R_{(i)(j)(i)(j)}^{(r)}-\left(K_{(i)}\right)_{b}^{a}\left(K_{(i)}\right)_{a}^{b}\right) \\
& \quad+O\left((1-\alpha)^{2}\right),
\end{aligned}
$$

where $\left(R^{(\alpha)}\right)_{\nu \sigma \lambda}^{\mu}$ denotes the bulk Riemann tensor evaluated on the orbifold, $\left(R^{(r)}\right)_{\nu \sigma \lambda}^{\mu}$ represents the regular part of the bulk Riemann tensor, $M_{4}$ refers to the regular manifold given in the $\alpha \rightarrow 1$ limit (where $2 \pi(1-\alpha)$ is the angular deficit of the cone), $G_{\mu \nu}$ corresponds to the bulk metric of the manifold, $\Sigma$ is the codimension- 2 surface located at the tip of the cone and given by the fixed-point set of the $Z_{n}$ symmetry of the orbifold, $\gamma_{a b}$ is the induced metric on $\Sigma$, $R_{(i)(j)(i)(j)}^{(r)}$ denotes the corresponding components of the Riemann tensor where $(i)$ and $(j)$ are the indices of the foliation $(i, j=1,2)$ and $\left(K_{(i)}\right)_{b}^{a}$ is the extrinsic curvature tensor of the surface $\Sigma$ with respect to the $i$ th direction of the foliation that is normal to the surface $(i=1,2)$, where a sum over repeated foliation indices is implied. We note that $\left(R^{(\alpha)}\right)_{\alpha \beta}^{\mu \nu}$ is divergent at $\Sigma$, and it can be written as

$$
\begin{aligned}
\left(R^{(\alpha)}\right)_{\sigma \lambda}^{\mu \nu}= & \left(R^{(r)}\right)_{\sigma \lambda}^{\mu \nu}+2 \pi(1-\alpha)\left(N_{\sigma \lambda}^{\mu \nu}+T_{\sigma \lambda}^{\mu \nu}\right) \delta_{\Sigma}, \\
N_{\rho \lambda}^{\mu \nu}= & {\left[\left(n_{(i)}\right)^{\mu}\left(n_{(i)}\right)_{\sigma}\left(n_{(j)}\right)^{\nu}\left(n_{(j)}\right)_{\lambda}\right.} \\
& \left.-\left(n_{(i)}\right)^{\mu}\left(n_{(i)}\right)_{\lambda}\left(n_{(j)}\right)^{\nu}\left(n_{(j)}\right)_{\sigma}\right],
\end{aligned}
$$

where $\delta_{\Sigma}$ is a codimension-2 delta function which only has support on $\Sigma,\left(n_{(i)}\right)_{\mu}$ is the $i$ th normal vector to the $\Sigma$ surface $(i=1,2)$ and $T_{\sigma \lambda}^{\mu \nu}$ is a tensor which depends on the extrinsic curvatures of $\Sigma$ with respect to the two directions of the foliation. In the case that the cone has a $U(1)$ rotational symmetry, $T_{\sigma \lambda}^{\mu \nu}=0$. However, in our case, although $T_{\sigma \lambda}^{\mu \nu}$ is left unspecified, it does encode the extrinsic curvature contributions to the quadratic terms.

Analogously, FPS obtained that for the square of the Ricci tensor,

$$
\begin{aligned}
& \int_{\hat{M}_{4}^{(\alpha)}} d^{4} x \sqrt{G}\left(R^{(\alpha)}\right)_{\mu \nu}\left(R^{(\alpha)}\right)^{\mu \nu} \\
& =\int_{M_{4}} d^{4} x \sqrt{G}\left(R^{(r)}\right)_{\mu \nu}\left(R^{(r)}\right)^{\mu \nu}+4 \pi(1-\alpha) \int_{\Sigma} d^{2} x \sqrt{\gamma}\left(R_{(i)(i)}^{(r)}\right. \\
& \left.\quad-\frac{1}{2}\left(K_{(i)}\right)_{a}^{a}\left(K_{(i)}\right)_{b}^{b}\right)+O\left((1-\alpha)^{2}\right),
\end{aligned}
$$

and for the square of the Ricci scalar,

$$
\begin{aligned}
& \int_{\hat{M}_{4}^{(\alpha)}} d^{4} x \sqrt{G}\left(R^{(\alpha)}\right)^{2} \\
& =\int_{M_{4}} d^{4} x \sqrt{G}\left(R^{(r)}\right)^{2}+8 \pi(1-\alpha) \\
& \quad \times \int_{\Sigma} d^{2} x \sqrt{\gamma}\left(R^{(r)}\right)+O\left((1-\alpha)^{2}\right) .
\end{aligned}
$$

Because, in the computation of $S_{\mathrm{EE}}$, we need to take the $\alpha \rightarrow 1$ limit, it is safe to neglect terms of quadratic or higher order in $(1-\alpha)$.

Finally, we have that the Gauss-Codazzi decomposition of the regular part of the Ricci scalar on $M_{4}$ gives

$$
\begin{aligned}
R^{(r)}= & -R_{(i)(j)(i)(j)}^{(r)}+2 R_{(i)(i)}^{(r)}+\mathcal{R}-\left(K_{(i)}\right)_{a}^{a}\left(K_{(i)}\right)_{b}^{b} \\
& +\left(K_{(i)}\right)_{a}^{b}\left(K_{(i)}\right)_{b}^{a},
\end{aligned}
$$

where we $\mathcal{R}$ is the intrinsic Ricci scalar at the surface $\Sigma$ (computed with the induced metric $\gamma_{a b}$ ), and the other quantities have the same meaning as for the quadratic terms presented above.

Now, considering Eqs. (10)-(14), we evaluate $\varepsilon_{4}$ on the $\hat{M}_{4}^{(\alpha)}$ orbifold. The Gauss-Bonnet term is given by

$$
\varepsilon_{4}=\sqrt{G} d^{4} x\left(R_{\nu \sigma \lambda}^{\mu} R_{\mu}^{\nu \sigma \lambda}-4 R_{\mu \nu} R^{\mu \nu}+R^{2}\right),
$$

and, therefore, we obtain that

$$
\int_{\hat{M}_{4}^{(\alpha)}} \varepsilon_{4}^{(\alpha)}=\int_{M_{4}} \varepsilon_{4}^{(r)}+8 \pi(1-\alpha) \int_{\Sigma} \varepsilon_{2}+O\left((1-\alpha)^{2}\right),
$$

where we used that $\varepsilon_{2}=\mathcal{R} \sqrt{\gamma} d^{2} x$ is the usual twodimensional Gauss-Bonnet term, which depends on the intrinsic Ricci scalar at the surface $\Sigma$. 
Furthermore, considering that (as shown in FPS) for squashed-cone manifolds in four dimensions, the Euler characteristic obeys the relation

$\chi_{4}\left(\hat{M}_{4}^{(\alpha)}\right)=\chi_{4}\left(M_{4}\right)+(1-\alpha) \chi_{2}(\Sigma)+O\left((1-\alpha)^{2}\right)$,

and also using Eq. (9) for the $m=1$ and $m=2$ cases, we obtain that the boundary terms (given by the corresponding Chern forms) are related by

$\int_{\partial \hat{M}_{4}^{(\alpha)}} B_{3}^{(\alpha)}=\int_{\partial M_{4}} B_{3}^{(r)}+8 \pi(1-\alpha) \int_{\partial \Sigma} B_{1}+O\left((1-\alpha)^{2}\right)$,

where $B_{1}$ is evaluated at the boundary of the codimension-2 surface $\Sigma$.

It is precisely this last relation which will be used in Sec. III, in order to evaluate the Euclidean action in the orbifold, which will ultimately give the expression for the renormalized EE when considering the renormalized Euclidean action which will be discussed in the following subsection.

\section{Renormalized Euclidean action and topological invariants}

In order to obtain a renormalized version of Eq. (8), to be able to compute the finite part of the $\mathrm{EE}$, we need to consider a suitably renormalized Euclidean action for the bulk gravity theory.

For AAdS spacetimes, there are different prescriptions for renormalizing the Euclidean on-shell action. The standard Holographic Renormalization method consists of adding counterterms to the action as surface terms, [11-17]. In doing so, the divergences occurring due to the presence of the infinite conformal factor in the metric at the boundary, as seen in its Fefferman-Graham expansion [6] are canceled out. The counterterms are functionals of the boundary metric, its intrinsic curvature and covariant derivatives thereof, in order to be consistent with a well-posed variational principle for the conformal class of spacetimes $\left[h_{i j}\right]$ at the boundary [16,17], after the Gibbons-Hawking-York term is included. Although there is a systematic procedure for computing the counterterms, in principle, at any order in the holographic radial coordinate $\rho$ and for any number of spacetime dimensions [12], the number of counterterms required grows rapidly with the dimension. Furthermore, the functional form of the terms in the series is different for different gravity actions including higher-curvature theories (e.g., Lovelock gravity theories).

The Kounterterms procedure, developed in Ref. [19], and further understood in Ref. [23], consists on adding a given boundary term to the AdS gravity action in order to both attain a well defined variational principle and to render the action finite. The particular term that is added is universal for all gravity theories of the Lovelock type and depends only on the number of dimensions of spacetime and on whether said number is odd or even. In the case of AAdS spacetimes in even dimensions, with $D=2 m$, the term added is the $m$ th Chern form [19]. For odd-dimensional spacetimes, the term added corresponds to the boundary term of the Chern-Simons transgression form of the AdS group [20]. In both cases, the added boundary term depends on both the intrinsic and extrinsic $\left(K_{j}^{i}\right)$ curvatures of the boundary in the radial foliation of the spacetime, and hence the name Kounterterms. Therefore, it is easy to particularize to the case of Fefferman-Graham expansion (with respect to the holographic radial coordinate $\rho$ ). In even-dimensional manifolds, there is a relation between the added boundary terms and topological terms. Indeed, the $m$ th Chern form is the boundary term associated with the Euler theorem, which relates the integral of the Euler term in the bulk with the Euler characteristic of the manifold, as mentioned in the previous subsection.

It is important to note that in $[22,23]$, it was proven that the addition of Kounterterms is equivalent to the standard Holographic Renormalization procedure in Einstein gravity in even dimmensions. As a matter of fact, standard counterterms are recovered when expressing these extrinsic counterterms in terms of the intrinsic curvature at the boundary (making extensive use of the FG expansion for $K_{j}^{i}$ ), order by order in the holographic radial coordinate $\rho$. However, the universality of the Kounterterms method with respect to different gravity theories (i.e., all theories of Lovelock type) and the fact that the closed-form expression for the boundary term is known for any dimension are its main practical advantages over the standard Holographic Renormalization procedure, but also its relation to topology is interesting on its own.

In this paper, we consider the renormalized action given by the Kounterterms prescription, which for the case of EH gravity in four-dimensional AAdS manifolds is given by [19]

$$
I_{E}^{\mathrm{ren}}=\frac{1}{16 \pi G}\left(\int_{M_{4}} d^{4} x \sqrt{G}(R-2 \Lambda)+\frac{\ell^{2}}{4} \int_{\partial M_{4}} B_{3}\right),
$$

where $\Lambda=-\frac{3}{\ell^{2}}$, and $B_{3}$ is given by

$$
B_{3}=-4 \int_{0}^{1} d t d^{3} x \sqrt{h} \delta_{\left[i_{1} i_{2} i_{3}\right]}^{\left[j_{1} j_{2} j_{3}\right]} K_{j_{1}}^{i_{1}}\left(\frac{1}{2} \mathcal{R}_{j_{2} j_{3}}^{i_{2} i_{3}}-t^{2} K_{j_{2}}^{i_{2}} K_{j_{3}}^{i_{3}}\right) .
$$

In Eq. (20), $h_{i j}$ is the metric at the boundary of spacetime, $\mathcal{R}_{i j}^{k \ell}$ is the Riemann curvature tensor at the boundary, computed with the $h_{i j}$ metric, and $K_{j}^{i}$ is the extrinsic curvature tensor of the boundary with respect to a radial foliation along the holographic radial coordinate $\rho$. 
The main reason for adopting this renormalization scheme is that the boundary term $B_{3}$ can be directly evaluated in the orbifold $\hat{M}_{4}^{(\alpha)}$ using the generalized Euler theorem for the boundary terms, as presented in Eq. (18). Then, the counterterm for the renormalized $\mathrm{EE}$ directly becomes the $B_{1}$ term evaluated at the entangling surface $\partial \Sigma$ as shown in Eq. (1). This will be explained in detail in the following section.

\section{RENORMALIZATION OF EE IN AdS 4 /CFT 3 THROUGH THE CHERN FORM}

After introducing the renormalized Euclidean action for the dual gravitational theory and also the generalization of the Euler theorem for four-dimensional squashed-cone manifolds, we proceed to compute the renormalized EE $\left(S_{\mathrm{EE}}^{\text {ren }}\right)$ by means of the replica trick. This is done by evaluating Eq. (8) using the renormalized gravitational action of Eq. (19). This assumes that if the gravitational action is itself renormalized, then the resulting EE will be renormalized as well.

The renormalized Euclidean on-shell action, evaluated on the conically singular manifold $\hat{M}_{4}^{(\alpha)}$, is given by

$$
I_{E}^{\mathrm{ren}}=\frac{1}{16 \pi G}\left(\int_{\hat{M}_{4}^{(\alpha)}} d^{4} x \sqrt{G}\left(R^{(\alpha)}-2 \Lambda\right)+\frac{\ell^{2}}{4} \int_{\partial \hat{M}_{4}^{(\alpha)}} B_{3}^{(\alpha)}\right) .
$$

As it was discussed by Lewkowicz and Maldacena [10] and by Dong [18], the Einstein-Hilbert part of Eq. (21) gives the usual RT area formula for the EE, when computing the derivative of Eq. (8) with respect to the conical angle parameter $\alpha$. Therefore, the counterterm that regularizes the EE will come from the $B_{3}^{(\alpha)}$ part. We define the counterterm of the Euclidean action as

$$
I_{E}^{c t}=\frac{\ell^{2}}{64 \pi G} \int_{\partial \hat{M}_{4}^{(\alpha)}} B_{3}^{(\alpha)},
$$

and, therefore, we proceed to compute the counterterm of the $\mathrm{EE}\left(S_{\mathrm{EE}}^{c t}\right)$ as

$$
S_{\mathrm{EE}}^{c t}=-\left.\partial_{\alpha} I_{E}^{c t}\left(\partial \hat{M}_{4}^{(\alpha)}\right)\right|_{\alpha=1},
$$

such that $S_{\mathrm{EE}}^{\mathrm{ren}}=S_{\mathrm{EE}}^{\mathrm{RT}}+S_{\mathrm{EE}}^{c t}$, where $S_{\mathrm{EE}}^{\mathrm{RT}}$ is the usual RT prescription for the EE.

Using Eq. (18) to evaluate $I_{E}^{c t}$, we have that

$$
S_{\mathrm{EE}}^{c t}=\frac{\ell^{2}}{8 G} \int_{\partial \Sigma} B_{1},
$$

and, therefore, we recover the expression for $S_{\mathrm{EE}}^{\text {ren }}$ given in Eq. (1).

In the next subsections, we will expand the integrands of $S_{\mathrm{EE}}^{\text {ren }}$ in their corresponding FG expansions, in order to verify the finiteness of the renormalized $\mathrm{EE}$, and also in order to show that our result is equivalent to the one obtained in [8]. We will also compute $S_{\mathrm{EE}}^{\text {ren }}$ for the particular case of a disclike entangling region in the three-dimensional CFT, with a global $\mathrm{AdS}_{4}$ gravitational dual (corresponding to the ground state of the CFT). In order to do this, we will consider the explicit embedding of the minimal surface $\Sigma$ and its boundary $\partial \Sigma$, as given in $[33,34]$ and as explained in what follows.

\section{A. Explicit covariant embedding}

Following the works by Hung, Myers and Smolkin [34], and by Schwimmer and Theisen [33] we consider that the embedding of the minimal surface $\Sigma$ on the bulk is given by

$$
\begin{aligned}
x^{i}\left(\tau, y^{a}\right) & =\left(x^{(0)}\right)^{i}\left(y^{a}\right)+\tau\left(x^{(2)}\right)^{i}\left(y^{a}\right)+\cdots \\
\left(x^{(2)}\right)^{i}\left(y^{a}\right) & =\frac{\ell^{2}}{2(d-2)} \kappa^{i}\left(y^{a}\right),
\end{aligned}
$$

where $\left\{\rho, x^{i}\right\}$ are bulk coordinates and $\left\{\tau, y^{a}\right\}$ are coordinates on the worldvolume of $\Sigma$.

We label the AAdS bulk metric as $G_{\mu \nu}$ and the metric at the spacetime boundary as $h_{i j}$. Analogously, we label the induced metric on $\Sigma$ as $\gamma_{a b}$ and the induced metric on its boundary $(\partial \Sigma)$ as $\tilde{\gamma}_{a b}$. As is well known (see, e.g., [7]), the metric $G_{\mu \nu}$ has a FG expansion [6] given by

$$
\begin{aligned}
d s_{G}^{2} & =G_{\mu \nu} d x^{\mu} d x^{\nu}=\frac{\ell^{2} d \rho^{2}}{4 \rho^{2}}+h_{i j}(\rho, x) d x^{i} d x^{j}, \\
h_{i j}(\rho, x) & =\frac{g_{i j}(\rho, x)}{\rho}, \\
g_{i j}(\rho, x) & =g_{i j}^{(0)}(x)+\rho g_{i j}^{(2)}(x)+\cdots,
\end{aligned}
$$

where $\rho$ is the holographic radial coordinate (the spacetime boundary is located at $\rho=0$ ).

Now, the induced metric $\gamma_{a b}$ is defined as

$$
\gamma_{a b}=\frac{\partial x^{\mu}}{\partial y^{a}} \frac{\partial x^{\nu}}{\partial y^{b}} G_{\mu \nu}
$$

and upon choosing the diffeomorphism gauge as $\tau=\rho$ and $\gamma_{a \tau}=0$, one obtains that

$$
\begin{aligned}
d s_{\gamma}^{2}= & \gamma_{a b} d y^{a} d y^{b}=\frac{\ell^{2}}{4 \tau^{2}}\left(1+\frac{\tau \ell^{2}}{(d-2)^{2}} \kappa^{i} \kappa^{j} g_{i j}^{(0)}+\cdots\right) d \tau^{2} \\
& +\tilde{\gamma}_{a b}(\tau, y) d y^{a} d y^{b} \\
\tilde{\gamma}_{a b}(\tau, y)= & \frac{\sigma_{a b}(\tau, y)}{\tau} \\
\sigma_{a b}(\tau, y)= & \sigma_{a b}^{(0)}(y)+\tau \sigma_{a b}^{(2)}(y)+\cdots
\end{aligned}
$$


which has the form of a FG-like expansion for the induced metric on $\Sigma$. To make this last statement more precise, $\gamma_{a b}$ is given by the pullback onto $\Sigma$ of the $G_{\mu \nu}$ bulk metric in the FG gauge.

We now explain the meaning of the coefficients in the FG expansions of the previous equations. In particular, $d$ is the dimension of the boundary of spacetime $(d=3$ in our case), $g_{i j}^{(0)}$ is the metric at the conformal boundary (where the three-dimensional CFT is defined) and $\sigma_{a b}^{(0)}$ is the induced metric on the entangling surface $\partial A$ (embedded in the conformal boundary) which is given by

$$
\sigma_{a b}^{(0)}=\frac{\partial\left(x^{(0)}\right)^{i}}{\partial y^{a}} \frac{\partial\left(x^{(0)}\right)^{j}}{\partial y^{b}} g_{i j}^{(0)} .
$$

Furthermore, $g_{i j}^{(2)}=-\ell^{2} S_{i j}^{(0)}$ where $S_{i j}^{(0)}$ is the Schouten tensor of the $g_{i j}^{(0)}$ metric given by

$$
\left(S^{(0)}\right)_{i j}=\frac{1}{(d-2)}\left(R_{i j}^{(0)}-\frac{g_{i j}^{(0)}}{2(d-1)} R^{(0)}\right),
$$

and $\sigma_{a b}^{(2)}$ is given by

$\sigma_{a b}^{(2)}=\frac{\partial\left(x^{(0)}\right)^{i}}{\partial y^{a}} \frac{\partial\left(x^{(0)}\right)^{j}}{\partial y^{b}} g_{i j}^{(2)}-\frac{\ell^{2}}{(d-2)} \kappa^{i} \kappa_{a b}^{j}\left(g^{(0)}\right)_{i j}$.

Finally,

$$
\kappa^{i}=\hat{n}_{(n)}^{i} \kappa_{a b}^{(n)}\left(\sigma^{(0)}\right)^{a b},
$$

where the extrinsic curvatures $\kappa_{a b}^{(n)}$ are defined with respect to the foliation that is normal to $\partial A$ (which is conformal to $\partial \Sigma$ ) embedded in the conformal boundary (which is conformal to the boundary of spacetime), and $\hat{n}_{(n)}^{i}$ are the vectors along the directions of the foliation $(n=1,2)$.

Having introduced the explicit covariant embedding of $\Sigma$ in $\hat{M}_{4}^{(\alpha)}$, and the corresponding FG expansions of $G_{\mu \nu}$ and $\gamma_{a b}$, we now proceed to show that $S_{\mathrm{EE}}^{\mathrm{ren}}$, as defined in Eq. (1), is finite and equivalent to the standard expression given in Eq. (2) and first obtained in [8].

\section{B. Proof of finiteness of $S_{\mathrm{EE}}^{\text {ren }}$}

With the previously considered embedding, we can check the cancellation of divergences in $S_{\mathrm{EE}}^{\text {ren }}$ for threedimensional CFTs with four-dimensional AAdS gravity duals. We note that the explicit value of $S_{\mathrm{EE}}^{\mathrm{ren}}$ depends on the shape of the entangling surface $\partial A$ at the conformal boundary. Here, we simply exhibit the divergences in $S_{\mathrm{EE}}^{\mathrm{RT}}$ (the standard Ryu-Takayanagi EE) and check that they are exactly cancelled by $S_{\mathrm{EE}}^{c t}$ [the EE counterterm given in Eq. (24)]. This cancellation is independent of the shape of $\partial A$.
In particular, we have

$$
\begin{aligned}
B_{1} & =-2 d y \sqrt{\tilde{\gamma}} \delta_{a}^{b} k_{b}^{a}, \\
S_{\mathrm{EE}}^{c t} & =-\frac{\ell^{2}}{4 G} \int_{\partial \Sigma} d y \sqrt{\tilde{\gamma}} \tilde{\gamma}^{a b} k_{a b}, \\
S_{\mathrm{EE}}^{\mathrm{RT}} & =\frac{1}{4 G} \int_{\Sigma} d^{2} y \sqrt{\gamma},
\end{aligned}
$$

where $k_{a b}$ is the extrinsic curvature of $\partial \Sigma$ with respect to the radial foliation along the holographic radial coordinate $\rho$ (not to be confused with $\kappa_{a b}^{(n)}$ for $\partial A$, or with $K_{i j}$ for $B$ ).

Now, we consider the FG expansion of each of the pieces. From Eq. (28), we have that the square root of the determinant of the metric on $\Sigma$ and on $\partial \Sigma$ are given, respectively, by

$$
\begin{aligned}
& \sqrt{\gamma}=\frac{\ell \sqrt{\sigma^{(0)}}}{2 \rho^{d / 2}}\left(1+\rho\left[\frac{\ell^{2}}{2(d-2)^{2}} \kappa^{i} \kappa^{j} g_{i j}^{(0)}+\frac{1}{2} \operatorname{tr}\left[\sigma^{(2)}\right]\right]+\cdots\right), \\
& \sqrt{\tilde{\gamma}}=\frac{\sqrt{\sigma^{(0)}}}{\rho^{(d-2) / 2}}\left(1+\frac{\rho}{2} \operatorname{tr}\left[\sigma^{(2)}\right]+\cdots\right)
\end{aligned}
$$

where $\operatorname{tr}\left[\sigma^{(2)}\right]$ denotes the trace of the $\sigma_{a b}^{(2)}$ tensor, given in the paragraph following Eq. (28). Also, $\tilde{\gamma}^{a b}$ is the inverse of the induced metric on $\partial \Sigma$, and it is given by $\tilde{\gamma}^{a b}=\rho\left(\left(\sigma^{(0)}\right)^{a b}-\rho\left(\sigma^{(2)}\right)^{a b}+\cdots\right)$. Now, considering the FG-like expansion of the induced metric on $\Sigma$, the extrinsic curvature $k_{a b}$ with respect to the radial foliation is computed, by definition, as $k_{a b}=\frac{-1}{2 \sqrt{\gamma_{\rho \rho}}} \partial_{\rho} \tilde{\gamma}_{a b}$. Thus, we have that

$$
k_{a b}=\frac{\sigma_{a b}^{(0)}}{\ell \rho}\left(1-\frac{\rho \ell^{2}}{2(d-2)^{2}} \kappa^{i} \kappa^{j} g_{i j}^{(0)}+\cdots\right) .
$$

Finally, in order to evaluate $S_{\mathrm{EE}}^{c t}$, we consider the expansion of $\sqrt{\tilde{\gamma}} \tilde{\gamma}^{a b} k_{a b}$ at the cutoff $\rho=\varepsilon$, where the limit of $\varepsilon \rightarrow 0$ has to be evaluated at the end. Thus, we have

$$
\begin{aligned}
\left.\sqrt{\tilde{\gamma}} \tilde{\gamma}^{a b} k_{a b}\right|_{\rho=\varepsilon}= & \frac{(d-2) \sqrt{\sigma^{(0)}}}{\ell \varepsilon^{(d-2) / 2}}\left(1+\varepsilon\left[\frac{(d-4)}{2(d-2)} \operatorname{tr}\left[\sigma^{(2)}\right]\right.\right. \\
& \left.\left.-\frac{\ell^{2}}{2(d-2)^{2}} \kappa^{i} \kappa^{j} g_{i j}^{(0)}\right]+\cdots\right) .
\end{aligned}
$$

Now we have all the pieces required to check that the divergences of $S_{\mathrm{EE}}^{\text {ren }}$ vanish. We, therefore, consider that

$$
S_{\mathrm{EE}}^{\mathrm{RT}}=\frac{1}{4 G} \int_{\Sigma} d^{2} y \sqrt{\gamma}=\frac{1}{4 G} \int_{\partial \Sigma_{\varepsilon}} d y \int_{\varepsilon}^{\rho_{\max }} d \rho \sqrt{\gamma},
$$

where $\rho_{\max }$ is the maximum value of $\rho$ in the $\Sigma$ minimal surface, which depends on the choice of entangling surface at the conformal boundary. By subsuming the finite part of the $\rho$ integral in a constant $C$, we can therefore write that 


$$
\begin{aligned}
\int_{\varepsilon}^{\rho_{\max }} d \rho \sqrt{\gamma}= & C+\frac{\ell \sqrt{\sigma^{(0)}}}{(d-2) \varepsilon^{(d-2) / 2}}\left(1+\varepsilon\left[\frac{(d-2)}{2(d-4)} \operatorname{tr}\left[\sigma^{(2)}\right]\right.\right. \\
& \left.\left.+\frac{\ell^{2}}{2(d-4)(d-2)} \kappa^{i} \kappa^{j} g_{i j}^{(0)}\right]+\cdots\right)
\end{aligned}
$$

And in our particular case, for three-dimensional CFTs, we note that only the leading terms in the $\varepsilon$ expansion contribute to the divergences. Thus, we have

$\left.\sqrt{\tilde{\gamma}} \tilde{\gamma}^{a b} k_{a b}\right|_{\rho=\varepsilon}=\frac{\sqrt{\sigma^{(0)}}}{\ell \varepsilon^{1 / 2}} ; \quad \int_{\varepsilon}^{\rho_{\max }} d \rho \sqrt{\gamma}=C+\frac{\ell \sqrt{\sigma^{(0)}}}{\varepsilon^{1 / 2}}$,

and, therefore, the structure of divergences of $S_{\mathrm{EE}}^{\text {ren }}$, in the limit of $\varepsilon \rightarrow 0$, give the following expression,

$S_{\mathrm{EE}}^{\mathrm{ren}}=\lim _{\varepsilon \rightarrow 0} \underbrace{\frac{1}{4 G} \int_{\partial \Sigma_{\varepsilon}} d y \frac{\ell \sqrt{\sigma^{(0)}}}{\varepsilon^{1 / 2}}-\frac{\ell^{2}}{4 G} \int_{\partial \Sigma_{\varepsilon}} d y \frac{\sqrt{\sigma^{(0)}}}{\ell \varepsilon^{1 / 2}}}_{0}+\frac{C}{4 G}$,

where $\frac{C}{4 G}$ is $O(1)$. We have therefore verified, explicitly, that the divergences in $S_{\mathrm{EE}}^{\mathrm{ren}}$ cancel each other for any entangling surface $\partial A$, and thus, $S_{\mathrm{EE}}^{\text {ren }}$ is correctly renormalized.

Finally, we show that our expression for $S_{\mathrm{EE}}^{\mathrm{ren}}$ [exhibited in Eq. (1)] is equivalent to the expression obtained in [8] and presented in Eq. (2). To see this, we consider that

$$
\begin{aligned}
k_{b}^{a} & =\tilde{\gamma}^{a c} k_{c b} \\
& =\frac{1}{\ell}\left(\delta_{b}^{a}-\rho\left[\left(\sigma^{(2)}\right)_{b}^{a}+\frac{\ell^{2}}{2(d-2)^{2}} \kappa^{i} \kappa^{j} g_{i j}^{(0)} \delta_{b}^{a}\right]+\cdots\right),
\end{aligned}
$$

and, therefore, for $d=3$, we have that

$$
\begin{aligned}
\left.B_{1}\right|_{\varepsilon} & =-\left.2 d y \sqrt{\tilde{\gamma}}\right|_{\varepsilon} \delta_{a}^{b} k_{b}^{a}=-\frac{2}{\ell} d y \sqrt{\left.\tilde{\gamma}\right|_{\varepsilon}}(\underbrace{\delta_{a}^{a}}_{1}+O(\varepsilon)), \\
S_{\mathrm{EE}}^{\mathrm{ren}} & =\frac{\operatorname{Vol}(\Sigma)}{4 G}-\frac{\ell}{4 G} \int_{\partial \Sigma} d y \sqrt{\tilde{\gamma}},
\end{aligned}
$$

thus recovering the known result.

\section{Topological interpretation of the renormalized EE}

We now give a topological interpretation of $S_{\mathrm{EE}}^{\text {ren }}$, considering the Euler theorem given in Eq. (9), and the definition of the curvature for the AdS group, which for an AAdS manifold is given by

$$
\left(\mathcal{F}_{\mathrm{AdS}}\right)_{\nu_{1} \nu_{2}}^{\mu_{1} \mu_{2}}=R_{\nu_{1} \nu_{2}}^{\mu_{1} \mu_{2}}+\frac{1}{\ell^{2}} \delta_{\left[\nu_{1} \nu_{2}\right]}^{\left[\mu_{1} \mu_{2}\right]}
$$

where $R_{\nu_{1} \nu_{2}}^{\mu_{1} \mu_{2}}$ is the Riemann tensor of the manifold.

Using Eq. (9), the Chern form that plays the role of counterterm for EE can be expressed as

$$
\int_{\partial \Sigma} B_{1}=\int_{\Sigma} d^{2} y \sqrt{\gamma} \mathcal{R}-\frac{\ell^{2}}{8 G}(4 \pi \chi(\Sigma)),
$$

where $\mathcal{R}$ is the Ricci scalar for the induced metric $\gamma_{a b}$ on $\Sigma$. Therefore, we can write $S_{\mathrm{EE}}^{\mathrm{ren}}$ as

$S_{\mathrm{EE}}^{\mathrm{ren}}=\frac{1}{4 G} \int_{\Sigma} d^{2} y \sqrt{\gamma}+\frac{\ell^{2}}{8 G} \int_{\Sigma} d^{2} y \sqrt{\gamma} \mathcal{R}-\frac{\ell^{2}}{8 G}(4 \pi \chi(\Sigma))$.

The above formula can be rewritten as

$$
S_{\mathrm{EE}}^{\mathrm{ren}}=\frac{\ell^{2}}{8 G} \int_{\Sigma} d^{2} y \sqrt{\gamma}\left(\mathcal{R}+\frac{2}{\ell^{2}}\right)-\frac{\pi \ell^{2}}{2 G} \chi(\Sigma),
$$

and using the properties of the totally antisymmetric Kronecker delta, we obtain

$S_{\mathrm{EE}}^{\mathrm{ren}}=\frac{\ell^{2}}{16 G} \int_{\Sigma} d^{2} y \sqrt{\gamma} \delta_{\left[a_{1} a_{2}\right]}^{\left.b_{1} b_{2}\right]} \underbrace{\left(\mathcal{R}_{b_{1} b_{2}}^{a_{1} a_{2}}+\frac{1}{\ell^{2}} \delta_{\left[b_{1} b_{2}\right]}^{\left[a_{1} a_{2}\right]}\right)}_{=\left(\left.\mathcal{F}_{\mathrm{AdS}}\right|_{\Sigma}\right)_{b_{1} b_{2}}^{a_{1} a_{2}}}-\frac{\pi \ell^{2}}{2 G} \chi(\Sigma)$.

The expression given in Eq. (47) is interesting because it makes manifest the connections of the renormalized EE with the topology of the extremal surface $\Sigma$, and also to its algebraic-geometrical properties as an AAdS Riemannian submanifold. In particular, we can recognize the curvature of the AdS group [35] for $\Sigma$, denoted $\left(\left.\mathcal{F}_{\text {AdS }}\right|_{\Sigma}\right)_{b_{1} b_{2}}^{a_{1} a_{2}}$, and also its Euler characteristic $\chi(\Sigma)$.

\section{Explicit example: Disclike entangling region in $\mathrm{CFT}_{3}$, with a global $\mathrm{AdS}_{4}$ bulk}

We now compute $S_{\mathrm{EE}}^{\text {ren }}$ for the particular case of a disclike entangling region in the ground state of a three-dimensional CFT, which is dual to a global $\mathrm{AdS}_{4}$ bulk on the gravity side, using our topological interpretation of the renormalized EE given in Eq. (47). The importance of this example is explained in detail in Sec. IV, but here we only mention that $S_{\mathrm{EE}}^{\text {ren }}$ is related to the $F$ quantity [36] by $S_{\mathrm{EE}}^{\text {ren }}=-F$ and to the $a$-charge [37] by $S_{\mathrm{EE}}^{\mathrm{ren}}=-2 \pi a_{3}$. Both of these order parameters of the CFT that are conjectured to decrease along RG flows between conformal fixed points (and can 
be thought of as generalizations of Zamolodchikov's c-theorem [38]).

We start by considering the global $\mathrm{AdS}_{4}$ bulk metric, which can be written in polar coordinates as

$d s_{G}^{2}=\frac{\ell^{2} d \rho^{2}}{4 \rho^{2}}+\frac{1}{\rho}\left(-d t^{2}+d r^{2}+r^{2} d \phi^{2}\right)=G_{\mu \nu} d x^{\mu} d x^{\nu}$.

Then, as is shown in Appendix B, the minimal surface in the bulk, which has a boundary that is conformal to the circle which constitutes the entangling surface, is given by the parametrization:

$$
\Sigma:\left\{t=\text { const } ; r^{2}+\ell^{2} \rho=R^{2}\right\},
$$

where $R$ is the radius of the circle. Now, we compute the induced metric on $\Sigma$, defined in Eq. (27), considering that the coordinates on $\Sigma$ are $y^{a}=\{\rho, \phi\}$, and those in the bulk are given by $x^{\mu}=\{\rho, t, r, \phi\}$. Then, for the induced metric on $\Sigma$, we obtain

$$
\begin{aligned}
d s_{\gamma}^{2} & =\frac{\ell^{2}}{4 \rho^{2}}\left(1+\frac{\ell^{2} \rho}{\left(R^{2}-\ell^{2} \rho\right)}\right) d \rho^{2}+\frac{\left(R^{2}-\ell^{2} \rho\right)}{\rho} d \phi^{2} \\
& =\gamma_{\alpha \beta} d y^{\alpha} d y^{\beta} .
\end{aligned}
$$

Given the induced metric on $\Sigma$, we compute its AdS curvature $\left(\left.\mathcal{F}_{\mathrm{AdS}}\right|_{\Sigma}\right)_{b_{1} b_{2}}^{a_{1} a_{2}}$ according to Eq. (43), and we find that it vanishes identically. Also, we note that $\Sigma$ is topologically equivalent to a disk, and therefore, $\chi(\Sigma)=1$. Thus, using the topological expression for $S_{\mathrm{EE}}^{\text {ren }}$ given in Eq. (47), we obtain

$$
S_{\mathrm{EE}}^{\mathrm{ren}}=-\frac{\pi \ell^{2}}{2 G},
$$

in agreement with the result obtained in [8].

Therefore, as further explained in Sec. IV, we have that for the three-dimensional CFT in the ground state, in terms of the quantities on the gravity side, $F=\frac{\ell^{2} \pi}{2 G_{4}}$ and $a_{3}=\frac{\ell^{2}}{4 G_{4}}$, in agreement with the previously known results. We mention however that in our computation we were able to exhibit new properties of the EE that, to the best of our knowledge, had not been noticed before.

\section{OUTLOOK}

So far, we have presented a new prescription for computing $S_{\mathrm{EE}}^{\text {ren }}$ in Eq. (1), which was derived directly from the replica trick by considering a suitably renormalized bulk gravity action [Eq. (19)]. We have also verified the finiteness of the EE obtained through such prescription, and its equivalence with the known result given in [8]. Furthermore, in Eq. (47), we have interpreted the result for
$S_{\mathrm{EE}}^{\text {ren }}$ in terms of the topological properties of the minimal surface $\Sigma$, and its geometrical properties as an AAdS submanifold.

EE, as considered in Quantum Information Theory for systems with finite dimensional Hilbert Spaces, is positive definite and can be computed directly as shown in Eq. (3). As it was mentioned in Sec. II A, it encodes the level of entanglement between a quantum subsystem $A$ and its complement $\left(A^{c}\right)$. In the case of CFTs (and more generally QFTs), the infinite-dimensional Hilbert space of the theory introduces the usual UV divergence in the EE. In the gravity side, this divergence appears in the area of the minimal surface $\Sigma$ due to the infinite conformal factor in the metric at the spacetime boundary. As it is explicitly shown in Sec. III D, the renormalized $\mathrm{EE}\left(S_{\mathrm{EE}}^{\mathrm{ren}}\right)$ is no longer positive definite, so its physical interpretation as an order parameter and its interest for the study of CFTs needs to be explicitated.

In particular, as mentioned in [8], the renormalized entanglement entropy $S_{\mathrm{EE}}^{\mathrm{ren}}$ is of interest because of its connection with quantities that are important for the study of Holographic Renormalization group (RG) flows. For example, in the case of a three-dimensional CFT at the boundary and a disc-shaped entangling region, $S_{\mathrm{EE}}^{\mathrm{ren}}=-F$, where the F quantity is defined in terms of the renormalized partition function of the theory on a three sphere as $F=-\ln Z_{S^{3}}$, and it decreases along RG flows [36]. Also, the renormalized EE for three-dimensional CFTs with a disc-shaped entangling region can be written in terms of the $a$-charge of the CFT as $S_{\mathrm{EE}}^{\text {ren }}=-2 \pi a_{3}$, where $a_{3}$ is conjectured to satisfy the relation that $\left(a_{3}\right)_{\mathrm{UV}} \geq\left(a_{3}\right)_{\mathrm{IR}}$, for any RG flow between conformal fixed points, as discussed in [37]. Therefore with our method, we recover the known results [8] of $F=\frac{\ell^{2} \pi}{2 G_{4}}$ and $a_{3}=\frac{\ell^{2}}{4 G_{4}}$, which can be translated in terms of the CFT quantities using the standard holographic dictionary $\left(G_{4}\right.$ is the gravitational constant of the four-dimensional AAdS bulk). All these quantities that (are conjectured to) decrease along RG flows can be considered as generalizations of Zamolodchikov's c-theorem [38]. They encode information about the number of degrees of freedom, which decreases as the theory flows to the infrared (IR).

Another quantity that is related to the $\mathrm{EE}$ and is useful for characterizing the informational content of CFTs is the mutual information (MI) $[39,40]$, which is defined in terms of differences of EEs as

$$
I_{A, B}=S_{\mathrm{EE}}(A)+S_{\mathrm{EE}}(B)-S_{\mathrm{EE}}(A \cup B),
$$

where $I_{A, B}$ denotes the MI between regions $\mathrm{A}$ and $\mathrm{B}$, and $S_{\mathrm{EE}}(X)$ denotes the $\mathrm{EE}$ of the entangling region $\mathrm{X}$. If one instead considers $S_{\mathrm{EE}}^{\text {ren }}(X)$ as the EE for region $X$, the result of $I_{A, B}$ is left unchanged for regions that do not overlap. Therefore, the renormalized EE can be used in the computation of MI without changing its properties. 
In particular, even if $S_{\mathrm{EE}}^{\text {ren }}$ can be negative, $I_{A, B}$ is always positive definite. This is important because it is usually the MI that is used when characterizing the amount of correlation between different regions in a CFT. For example, the MI can be used to place bounds on correlators of operators defined on separate regions [40].

As it will be described in a follow-up paper, we can extend the method for computing $S_{\mathrm{EE}}^{\text {ren }}$ to AAdS manifolds of arbitrary even dimensions by considering the renormalized Euclidean action given in [19], and by repeating the replica procedure. As future work, we will also study how to extend the scheme to AAdS manifolds of arbitrary odd dimensions, considering the renormalized Euclidean gravitational action discussed in [20]; and also to higher-curvature theories of gravity, specially those of the Lovelock class $[41,42]$.

We will also study the application of our renormalization procedure to other QIT measures, like the entanglement Renyi entropies (EREs) [18,43-45] and the complexity [4649], for CFTs of arbitrary dimensions with AAdS gravity duals. Regarding the complexity, we mention that an example of topological renormalization has already been achieved in [49], although only for the particular case of $\mathrm{AdS}_{3} / \mathrm{CFT}_{2}$.

\section{ACKNOWLEDGMENTS}

The authors thank Y. Novoa for interesting discussions. G. A. is a Universidad Andres Bello (UNAB) Ph.D. Scholarship holder, and his work is supported by Dirección General de Investigación (DGI-UNAB). This work is funded in part by Fondo Nacional de Desarrollo Científico y Tecnológico (FONDECYT) Grants No. 1170765 and No. 3180620, UNAB Grant No. DI-1336-16/R and Comisión Nacional de Investigación Científica y Tecnológica (CONICYT) Grant No. DPI 20140115.

\section{APPENDIX A: DERIVATION OF THE MINIMAL AREA CONDITION IN GLOBAL ADS}

In Sec. III D, we consider that the minimal surface $\Sigma$ in the global $\mathrm{AdS}_{4}$ bulk for a disclike entangling region in the dual three-dimensional CFT in its ground state is given by $\Sigma:\left\{t=\right.$ const; $\left.r^{2}+\ell^{2} \rho=R^{2}\right\}$, where $R$ is the radius of the disc. Here, we proceed to explicitly justify this claim. We first derive the minimal surface condition, in the form of an Euler-Lagrange differential equation that has to be obeyed by the embedding function of the minimal surface $\Sigma$, and then we proceed to check that the surface $\Sigma$ as defined above does indeed satisfy this condition. We note that this analysis is standard, and the reason why we repeat it here is because there is a small mistake in the treatment done by Taylor and Woodhead, presented in Eq. (3.12) of [8].

We first consider the metric of global $\operatorname{AdS}_{D}$, written in cartesian coordinates:

$d s_{G}^{2}=G_{\mu \nu} d x^{\mu} d x^{\nu}=\frac{\ell^{2} d \rho^{2}}{4 \rho^{2}}+\frac{-d t^{2}+\delta_{a b} d x^{a} d x^{b}}{\rho}$.
Then, we consider the parametrization of a codimension-2 surface $\Sigma$, with worldvolume coordinates given by $\tau$ and $y^{a}$ at $t=$ const. The embedding is done in the static gauge, such that $\rho=\tau$ and $x^{a}=y^{a}$, for $a=1, \ldots, D-3$, and $x^{D-2}=z\left(\rho, x^{a}\right)$, where $z$ is the embedding function and $D$ is the dimension of the bulk manifold. Then, the induced metric $\gamma_{a b}$ is given by $\gamma_{a b}=\frac{\partial x^{\mu}}{\partial y^{a}} \frac{\partial x^{\nu}}{\partial y^{b}} G_{\mu \nu}$, and in terms of the embedding function $z\left(\rho, x^{a}\right)$, we obtain

$$
\begin{aligned}
& \gamma_{\rho \rho}=G_{\rho \rho}+z_{, \rho} z_{, \rho} G_{z z}=\frac{\ell^{2}}{4 \rho^{2}}+\frac{z_{, \rho} z_{, \rho}}{\rho}, \\
& \gamma_{a b}=G_{a b}+z_{, a} z_{, b} G_{z z}=\frac{1}{\rho}\left(\delta_{a b}+z_{, a} z_{, b}\right), \\
& \gamma_{\rho a}=z_{, \rho} z_{, a} G_{z z}=\frac{z_{, \rho} z_{, a}}{\rho} .
\end{aligned}
$$

Now, we can derive the minimal area condition. In order to do this, we consider that $\operatorname{Vol}(\Sigma)=\int_{\Sigma} d^{D-2} y \sqrt{\gamma}$ is the area functional, and we define the auxiliary function $m\left(\rho, x^{a}\right)=\sqrt{1+4 \frac{\rho}{\ell^{2}} z_{, \rho} z_{, \rho}+z_{, a} z_{, a}}$, such that $\operatorname{Vol}(\Sigma)=$ $\int_{\Sigma} d^{D-2} y \frac{\ell m\left(\rho, x^{a}\right)}{2 \rho^{(D-1) / 2}}$. Then, we impose that the variation of the area functional with respect to the embedding function $z\left(\rho, x^{a}\right)$ has to be zero, in order for the surface $\Sigma$ to have extremal area. Therefore, the $z\left(\rho, x^{a}\right)$ corresponding to said minimal surface has to fulfill the differential equation resulting from the extremization condition. To derive the extremization condition, we consider that under the variation,

$$
\begin{aligned}
\delta_{z} \operatorname{Vol}(\Sigma)= & \int_{\Sigma} d^{D-2} y \frac{\ell}{4 m\left(\rho, x^{a}\right) \rho^{(D-1) / 2}} \\
& \times\left(8 \frac{\rho}{\ell^{2}} z_{, \rho} \delta z_{, \rho}+2 z_{, a} \delta z_{, a}\right),
\end{aligned}
$$

and then, requiring that $\delta_{z} \operatorname{Vol}(\Sigma)=0$ and integrating by parts, we obtain the corresponding Euler-Lagrange condition given by

$\partial_{a}\left(\frac{z_{, a}}{4 \rho^{(D-1) / 2} m\left(\rho, x^{a}\right)}\right)+\partial_{\rho}\left(\frac{z_{, \rho}}{\ell^{2} \rho^{(D-3) / 2} m\left(\rho, x^{a}\right)}\right)=0$.

Thus, in order for $\Sigma$ to be the minimal surface, its embedding function $z\left(\rho, x^{a}\right)$ has to satisfy Eq. (A4). To argue that $\Sigma$ is a minimum, and not a maximum, we note that due to the divergent conformal factor in the metric at $\rho \rightarrow 0$, the maximum is not well defined (intuitively, it would be a surface located entirely at the boundary of spacetime). Of course, there may be more than one surface $\Sigma$ that satisfies Eq. (A4), which would mean that these are multiple local minima of the area functional. In such a case, the true 
minimal surface is the one among them that has the smallest value for the area (after a suitable renormalization, by, for example, the method described in the body of this paper).

Now, in the next section, we verify that the surface $\Sigma$ of Sec. III D does indeed satisfy the extremal area condition of Eq. (A4).

\section{APPENDIX B: VERIFICATION THAT THE $\boldsymbol{\Sigma}$ CONSIDERED FOR A DISCLIKE ENTANGLING REGION IS THE MINIMAL SURFACE}

In the case of global $\mathrm{AdS}_{4}$, we consider a surface $\Sigma$ parametrized as $\Sigma:\left\{t=\right.$ const; $\left.r^{2}+\ell^{2} \rho=R^{2}\right\}$, and we proceed to show that it satisfies the extremal area condition of Eq. (A4). We first write the corresponding embedding function $z(\rho, x)$ in cartesian coordinates, considering that $r^{2}=x^{2}+z^{2}$. Thus we have that $z(\rho, x)=$ $\pm \sqrt{R^{2}-\ell^{2} \rho-x^{2}}$. Then, computing the derivatives of the embedding function, we have that

$$
\begin{aligned}
z_{, a} & =\mp \frac{x}{\sqrt{R^{2}-\ell^{2} \rho-x^{2}}} ; \quad z_{, \rho}=\mp \frac{\ell^{2}}{2 \sqrt{R^{2}-\ell^{2} \rho-x^{2}}}, \\
m(\rho, x) & =\sqrt{1+4 \frac{\rho}{\ell^{2}} z_{, \rho} z_{, \rho}+z_{, a} z_{, a}}=\frac{R}{\sqrt{R^{2}-\ell^{2} \rho-x^{2}}},
\end{aligned}
$$

and replacing the corresponding terms into Eq. (A4), we have that

$$
\begin{aligned}
& \partial_{a}\left(\frac{z_{, a}}{4 \rho^{(D-1) / 2} m\left(\rho, x^{a}\right)}\right)+\partial_{\rho}\left(\frac{z_{, \rho}}{\ell^{2} \rho^{(D-3) / 2} m\left(\rho, x^{a}\right)}\right) \\
& \quad= \pm\left(-\frac{1}{2 R} \partial_{\rho}\left(\frac{1}{\rho^{1 / 2}}\right)-\frac{1}{4 R \rho^{3 / 2}}\right)=0,
\end{aligned}
$$

and therefore, $\Sigma$ is indeed the minimal surface.
[1] J. M. Maldacena, Adv. Theor. Math. Phys. 2, 231 (1998); Int. J. Theor. Phys. 38, 1113 (1999).

[2] S. S. Gubser, I. R. Klebanov, and A. M. Polyakov, Phys. Lett. B 428, 105 (1998).

[3] E. Witten, Adv. Theor. Math. Phys. 2, 253 (1998).

[4] S. Ryu and T. Takayanagi, Phys. Rev. Lett. 96, 181602 (2006).

[5] M. Rangamani and T. Takayanagi, in Holographic Entanglement Entropy, Lecture Notes in Physics (Springer, Cham, 2017).

[6] C. Fefferman and C. R. Graham, in The mathematical heritage of Elie Cartan (Lyon 1984), Astérisque/Numero Hors Serie (CNRS, Paris, 1985), p. 95.

[7] C. Imbimbo, A. Schwimmer, S. Theisen, and S. Yankielowicz, Classical Quantum Gravity 17, 1129 (2000).

[8] M. Taylor and W. Woodhead, J. High Energy Phys. 08 (2016) 165.

[9] X. Dong, J. High Energy Phys. 01 (2014) 044.

[10] A. Lewkowycz and J. Maldacena, J. High Energy Phys. 08 (2013) 090.

[11] R. Emparan, C. V. Johnson, and R. C. Myers, Phys. Rev. D 60, 104001 (1999).

[12] P. Kraus, F. Larsen, and R. Siebelink, Nucl. Phys. B563, 259 (1999).

[13] S. de Haro, K. Skenderis, and S. N. Solodukhin, Commun. Math. Phys. 217, 595 (2001).

[14] V. Balasubramanian and P. Kraus, Commun. Math. Phys. 208, 413 (1999).

[15] M. Henningson and K. Skenderis, J. High Energy Phys. 07 (1998) 023.

[16] I. Papadimitriou and K. Skenderis, IRMA Lect. Math. Theor. Phys. 8, 73 (2005).
[17] I. Papadimitriou and K. Skenderis, J. High Energy Phys. 08 (2005) 004.

[18] X. Dong, Nat. Commun. 7, 12472 (2016).

[19] R. Olea, J. High Energy Phys. 06 (2005) 023.

[20] R. Olea, J. High Energy Phys. 04 (2007) 073.

[21] G. Kofinas and R. Olea, J. High Energy Phys. 11 (2007) 069.

[22] O. Miskovic, R. Olea, and M. Tsoukalas, J. High Energy Phys. 08 (2014) 108.

[23] O. Miskovic and R. Olea, Phys. Rev. D 79, 124020 (2009).

[24] D. V. Fursaev and S. N. Solodukhin, Phys. Rev. D 52, 2133 (1995).

[25] D. V. Fursaev, A. Patrushev, and S. N. Solodukhin, Phys. Rev. D 88, 044054 (2013).

[26] H. Casini, M. Huerta, and R. C. Myers, J. High Energy Phys. 05 (2011) 036.

[27] L. Y. Hung, R. C. Myers, and M. Smolkin, J. High Energy Phys. 04 (2011) 025.

[28] J. D. Bekenstein, Phys. Rev. D 7, 2333 (1973).

[29] J. M. Bardeen, B. Carter, and S. Hawking, Commun. Math. Phys. 31, 161 (1973).

[30] S. Hawking, Commun. Math. Phys. 43, 199 (1975).

[31] R. B. Mann and S. N. Solodukhin, Phys. Rev. D 54, 3932 (1996).

[32] F. Dahia and C. Romero, Mod. Phys. Lett. A 14, 1879 (1999).

[33] A. Schwimmer and S. Theisen, Nucl. Phys. B801, 1 (2008).

[34] L. Hung, R. C. Myers, and M. Smolkin, J. High Energy Phys. 04 (2011) 025.

[35] P. Mora, R. Olea, R. Troncoso, and J. Zanelli, J. High Energy Phys. 02 (2006) 067.

[36] D. L. Jafferis, I. R. Klebanov, S. S. Pufu, and B. R. Safdi, J. High Energy Phys. 06 (2011) 102. 
[37] R. C. Myers and A. Sinha, Phys. Rev. D 82, 046006 (2010).

[38] A. B. Zamolodchikov, Pis'ma Zh. Eksp. Teor. Fiz. 43, 565 (1986) [JETP Lett. 43, 730 (1986)].

[39] B. Swingle, arXiv:1010.4038.

[40] M. M. Wolf, F. Verstraete, M. B. Hastings, and J. I. Cirac, Phys. Rev. Lett. 100, 070502 (2008).

[41] D. Lovelock, J. Math. Phys. (N.Y.) 12, 498 (1971).

[42] D. Lovelock, J. Math. Phys. (N.Y.) 13, 874 (1972).

[43] M. Headrick, Phys. Rev. D 82, 126010 (2010).
[44] J. C. Baez, arXiv:1102.2098.

[45] J. Hung, R. C. Myers, M. Smolkin, and A. Yale, J. High Energy Phys. 12 (2011) 047.

[46] M. Alishahiha, Phys. Rev. D 92, 126009 (2015).

[47] D. Stanford and L. Susskind, Phys. Rev. D 90, 126007 (2014).

[48] A. R. Brown, D. A. Roberts, L. Susskind, B. Swingle, and Y. Zhao, Phys. Rev. Lett. 116, 191301 (2016).

[49] R. Abt, J. Erdmenger, H. Hinrichsen, C. M. MelbyThompson, R. Meyer, C. Northe, and I. A. Reyes, arXiv: 1710.01327. 